\title{
Prostatic abscess with infected aneurysms and spondylodiscitis after transrectal ultrasound-guided prostate biopsy: a case report and literature review
}

\author{
Shunichiro Nomura* ${ }^{*}$, Yuka Toyama, Jun Akatsuka, Yuki Endo, Ryoji Kimata, Yasutomo Suzuki,
}

Tsutomu Hamasaki, Go Kimura and Yukihiro Kondo

\begin{abstract}
Background: Transrectal ultrasonography (TRUS)-guided prostate biopsy is the conventional method of diagnosing prostate cancer. TRUS-guided prostate biopsy can occasionally be associated with severe complications. Here, we report the first case of a prostate abscess with aneurysms and spondylodiscitis as a complication of TRUS-guided prostate biopsy, and we review the relevant literature.

Case presentation: A 78-year-old man presented with back pain, sepsis, and prostate abscesses. Twenty days after TRUS-guided prostate biopsy, he was found to have a 20-mm diameter abdominal aortic aneurysm that expanded to $28.2 \mathrm{~mm}$ in the space of a week, despite antibiotic therapy. Therefore, he underwent transurethral resection of the prostate to control prostatic abscesses. Although his aneurysm decreased to $23 \mathrm{~mm}$ in size after surgery, he continued to experience back pain. He was diagnosed as having pyogenic spondylitis and this was managed using a lumbar corset. Sixty-four days after the prostate biopsy, the aneurysm had re-expanded to $30 \mathrm{~mm}$; therefore, we performed endovascular aneurysm repair (EVAR) using a microcore stent graft 82 days after the biopsy. Four days after the EVAR, the patient developed acute cholecystitis, and he underwent endoscopic retrograde biliary drainage. One hundred and sixty days after the prostate biopsy, all the complications had improved, and he was discharged. A literature review identified a further six cases of spondylodiscitis that had occurred after transrectal ultrasound-guided prostate biopsy.
\end{abstract}

Conclusions: We have reported the first case of a complication of TRUS-guided prostate biopsy that involved prostatic abscesses, aneurysms, and spondylodiscitis. Although such complications are uncommon, clinicians should be aware of the potential for such severe complications of this procedure to develop.

Keywords: Spondylodiscitis, Aneurysm, Prostate biopsy

\section{Background}

Transrectal ultrasonography (TRUS)-guided prostate biopsy is the standard method of diagnosing prostate cancer [1]. TRUS-guided prostate biopsy is a relatively

*Correspondence: s-nomura@nms.ac.jp

Department of Urology, Nippon Medical School, 1-1-5 Sendagi,

Bunkyo-ku, Tokyo 113-8603, Japan safe method that is usually well-tolerated by patients, although minor complications (such as pain, hematuria, hematospermia, and rectal hemorrhage) or, rarely, significant complications (such as sepsis, macroscopic hematoma, and urinary retention) can develop [2]. Here, we report the first case of prostatic abscess with aneurysms and spondylodiscitis after TURS-guided prostate biopsy, and contextualize this with a review of the literature. 


\section{Case presentation}

A 78-year-old man was hospitalized to undergo TRUSguided prostatic biopsy because he had a serum prostatespecific antigen (PSA) concentration of $15.86 \mathrm{ng} / \mathrm{ml}$. The TRUS-measured prostate volume was $41 \mathrm{ml}$. His medical history included coronary artery disease with previous intracoronary stenting. He received intravenous (IV) cefazolin $2 \mathrm{~g}$ daily as antimicrobial prophylaxis prior to the biopsy. The day after the biopsy, he had a fever (temperature $39.5{ }^{\circ} \mathrm{C}$ ), but his vital signs were otherwise normal. Blood analyses revealed a white blood cell (WBC) count of $7100 / \mu \mathrm{l}$. After 3 days, he was started on intravenous ceftriaxone and gamma-globulin $5 \mathrm{~g}$ because of a continuous fever and a high serum $\mathrm{C}$-reactive protein (CRP) concentration (Fig. 1). After 5 days, a gram-negative bacillus was grown on blood culture. A digital rectal examination revealed an enlarged prostate. Computed tomography (CT) of the pelvis showed mild enlargement of the prostate. Therefore, the patient's antibiotics were changed to meropenem $1.5 \mathrm{~g}$ and clindamycin to treat the sepsis of prostatitis, and a transabdominal catheter was placed into his urinary bladder. However, his condition worsened 6 days later and he had difficulty breathing, with an oxygen saturation of $85 \%$, according to pulse oximetry, and a low pO2 (32.4 $\mathrm{mmHg})$, according to arterial blood gas measurement. A chest X-ray showed air-space consolidation. Therefore, he was transferred to the intensive care unit for the diagnosis of acute respiratory distress syndrome (ARDS).

At this time, a further blood culture showed the presence of extended-spectrum-lactamase (ESBL)-producing Escherichia coli (E. coli), which was sensitive to imipenem; therefore, his antibiotics were changed accordingly. Twelve days later, rectal examination revealed a tender, fluctuant prostate consistent with a prostatic abscess. The CT images were consistent with the presence of three prostatic abscesses $(<1.5 \mathrm{~cm}$ each). Thirteen days after the prostate biopsy, his bacteremia had improved and his ARDS had resolved; therefore, he was transferred to the general ward. Twenty days after the prostate biopsy, an abdominal/pelvic CT scan was performed to identify any remaining prostatic abscesses. However, instead, the scan revealed the presence of an abdominal aortic aneurysm with surrounding periaortic inflammatory changes (Fig. 2a). Therefore, IV doripenem was administered. CT examination after 26 days showed no change in the prostatic abscesses, but the aneurysm had expanded to a diameter of $28.2 \mathrm{~mm}$.

Transurethral resection of the prostate was planned as a means of controlling prostatic abscesses for day 27 after the prostate biopsy. During the surgery, a whitish purulent substance discharged from the prostate. After

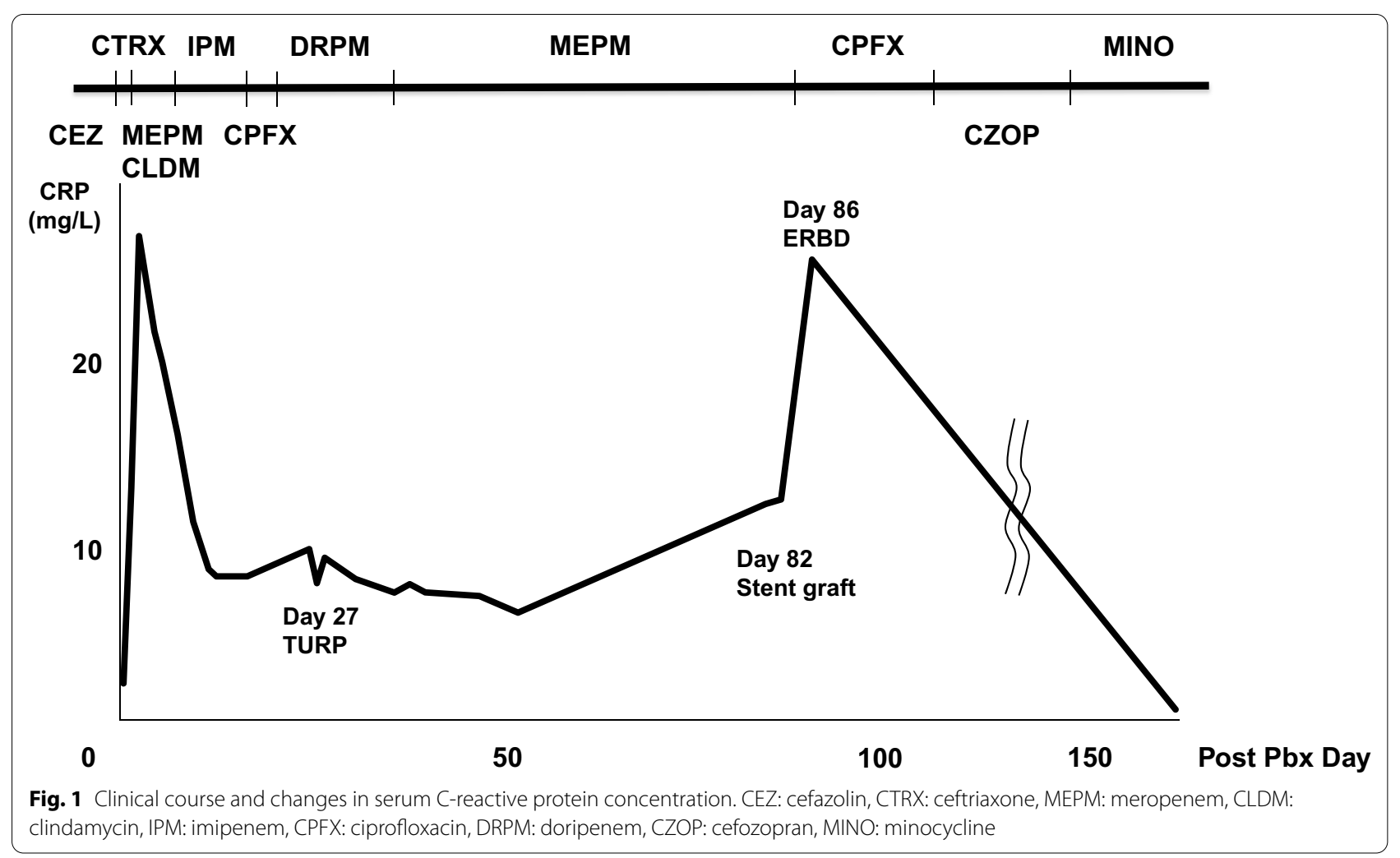



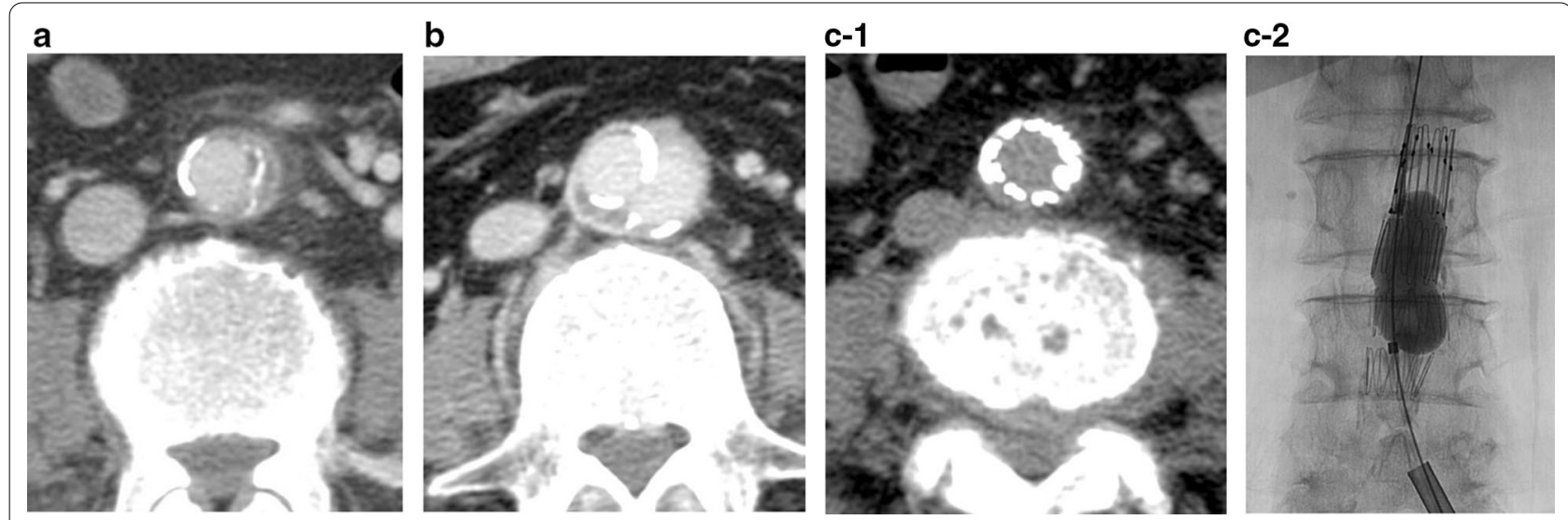

Fig. 2 Images of the aortic aneurysm. a Initial computed tomography (CT) image of the abdomen/pelvis, demonstrating a 20-mm abdominal aortic aneurysm, with evidence of inflammatory changes, 20 days after prostate biopsy. $\mathbf{b}$ Repeat CT image revealing that the abdominal aortic aneurysm diameter has increased to $30 \mathrm{~mm}, 64$ days after the prostate biopsy. c CT and angiography showing the successful exclusion of the aortic rupture by endovascular stent grafting

surgery, the patient made excellent progress. Postoperative CT (31 days after prostate biopsy) showed that the aneurysm had a satisfactory appearance $(23 \mathrm{~mm}$ diameter). However, he had also been experiencing back pain for 4 weeks, from day 11 after the prostate biopsy. Contrast-enhanced magnetic resonance imaging of the lumbar spine revealed contrast enhancement of the L2, L4, and L5 vertebral bodies (Fig. 3). Therefore, he was diagnosed as having pyogenic spondylitis and this was managed using a lumbar corset. Sixty-four days after the original biopsy, the aneurysm had re-expanded to $30 \mathrm{~mm}$ (Fig. 2b), but as the patient's condition was unsatisfactory, surgery was not attempted. Instead, he underwent endovascular aneurysm repair (EVAR) using a microcore stent graft at our hospital 82 days after the prostate biopsy (Fig. 2c). Four days after the endovascular treatment, he developed a stomach ache and CT revealed acute cholecystitis. Therefore, endoscopic retrograde biliary drainage (ERBD) was performed and the patient's antibiotic was changed to ciprofloxacin. By day 160 after the prostate biopsy, all the complications had improved, and the patient was discharged. He then underwent active surveillance for prostate cancer 1 year after the original diagnosis.

\section{Discussion and conclusions}

To the best of our knowledge, prostate abscess with infected aneurysms and spondylodiscitis has not been reported in the literature as a complication of transrectal prostate biopsy. Furthermore, infected aneurysm alone is an extremely rare complication of TURS-guided prostate biopsy: only two cases have been reported previously [3].

Infected aneurysms can arise because of the hematogenous spread of bacteria, which cause aneurysmal changes

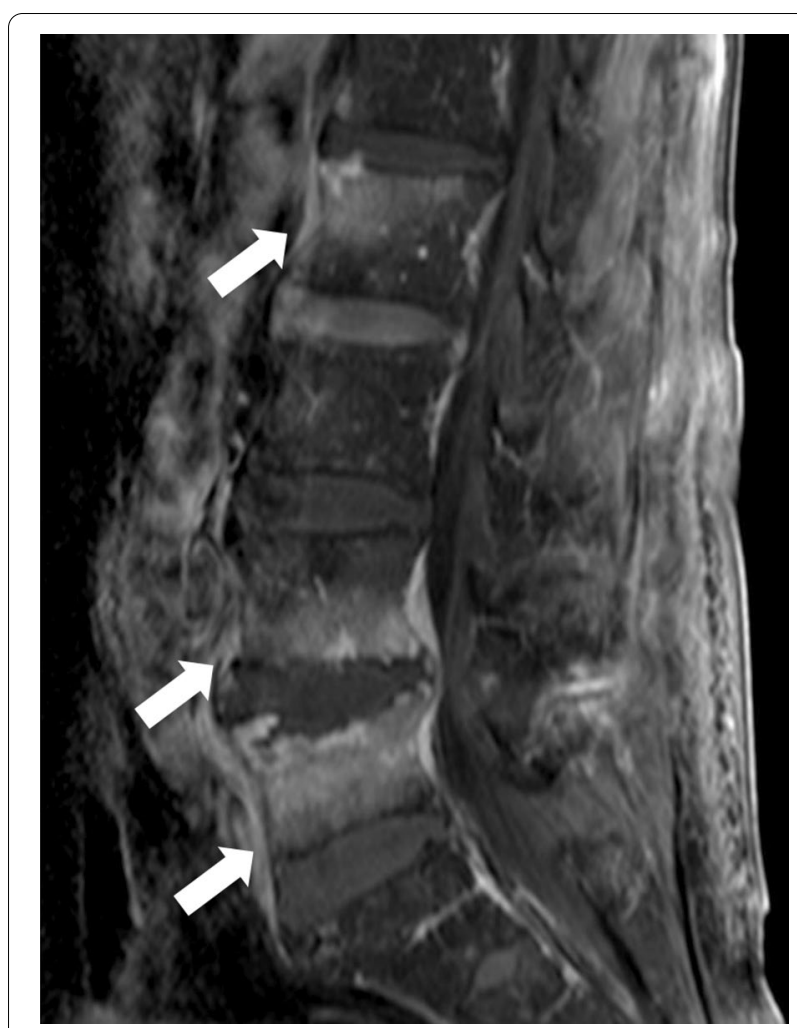

Fig. 3 Lumbar images obtained 40 days after prostate biopsy. Contrast-enhanced T1-weighted magnetic resonance image of the lumbar spine shows contrast-enhancement of the $L 2, L 4$, and $L 5$ vertebral bodies

in the arterial wall, and a pathogen can be further disseminated by this route to cause a prostate abscess. The gold-standard treatment of the disease remains surgical resection and debridement of the infected aorta, 


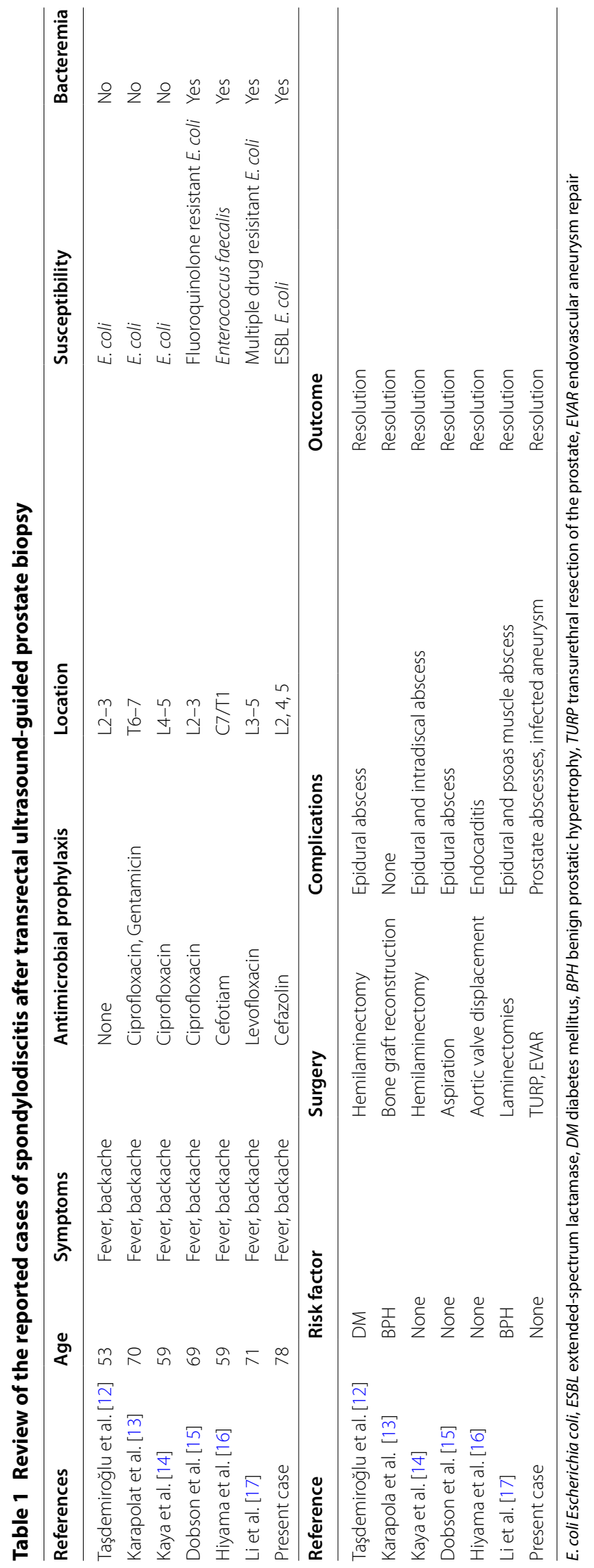


involving the placement of an interposition graft or extra-anatomic bypass, and long-term antibiotic therapy. However, this procedure is associated with high mortality of $13.3-40 \%[4,5]$. Therefore, EVAR has been developed as an elective open method of repair of an infected aneurysm, especially for high-risk surgical patients. Because the present patient was high-risk, we chose to treat the aneurysm using EVAR, and although he developed acute cholecystitis 4 days after the EVAR, his condition was improved by ERBD.

Spondylodiscitis is also an extremely rare complication of TURS-guided prostate biopsy. The incidence of spondylodiscitis ranges from 0.4 to 2.4/100,000 in Europe [6]. Six cases of spondylodiscitis following TRUS-guided prostate biopsy have been previously described in the English-speaking literature, (Table 1) and in all these cases the clinical symptoms were fever and backache. $E$. coli was the dominant cause of the spondylodiscitis, being identified in five of the cases, three of which were antibiotic-resistant. Enterococcus faecalis was identified as the causative agent in the remaining case. Here, we report the first case of spondylodiscitis that was caused by ESBL E. coli. Four of the seven reported cases had positive blood culture results. Lumbar vertebrae are most frequently affected in spondylodiscitis, as in five of the reported cases, including the present case. Risk factors to infection include immunosuppression, diabetes mellitus, and benign prostatic hypertrophy. Although three of the reported cases had risk factors, our patient had none of the risk factors. Five of the cases were treated using a surgical intervention, but the spondylodiscitis in the present case was treated by antibiotic therapy and spinal immobilization. Four of the cases also had an epidural abscess. However, all the identified lesions resolved, suggesting that such lesions have good prognoses.

Fluoroquinolones, and particularly ciprofloxacin, are widely used prophylactically alongside TRUS-guided prostate biopsy. However, the incidence of infectious complications associated with prostate biopsy has significantly increased in recent years [7]. Recent studies have shown that approximately half of post-biopsy infections are resistant to fluoroquinolone, and many are also resistant to other antibiotics [8]. Therefore, to prevent postbiopsy infection, we should consider selectively targeting antibiotic prophylaxis by performing a pre-biopsy rectal culture. Taylor et al. reported that targeted antimicrobial prophylaxis is associated with a notable reduction, in the incidence of infectious complications associated with TRUS-guided prostate biopsy that were caused by fluoroquinolone-resistant organisms, as well as a reduction in the overall cost of care. No infectious complications arose in the 112 men who received targeted antimicrobial prophylaxis, whereas there were nine cases (including one of sepsis) among the 345 men who were on empirical therapy [9]. Other proposed procedures include transperineal prostate biopsy and selectively augmented prophylaxis with two antibiotics in higher risk patients $[10,11]$.

In conclusion, we have reported the first case of prostatic abscess with aneurysm and spondylodiscitis after TRUS-guided prostate biopsy. Although this combination of complications is extremely rare, we should be aware of the possibility that severe complications can arise following this procedure.

\section{Abbreviations}

TRUS: Transrectal ultrasonography; IV: Intravenous; CRP: C-reactive protein; CT: Computed tomography; EVAR: Endovascular aneurysm repair.

\section{Acknowledgements}

We thank Mark Cleasby, PhD, from Edanz Group (https://en-author-services. edanzgroup.com/ac) for editing a draft of this manuscript. We also thank Megumi Nomura for your support.

\section{Authors' contributions}

SN contributed to the acquisition of the history, performed the imaging, and wrote the manuscript. YT and JA administered the treatment. YE and RK reviewed the manuscript and gave their opinions. YS supervised the research and helped write the manuscript. TH revised the manuscript. GK reviewed the manuscript. YK acted as a supervisor and reviewed the manuscript. All the authors read and approved the final manuscript.

\section{Funding}

None.

\section{Availability of data and materials}

Records and data pertaining to this case are held in the patient's secure medical records in Nippon Medical School Hospital.

\section{Ethics approval and consent to participate}

This ethics committee of Nippon Medical School Hospital approved the study, and the patient gave his written informed consent for the analysis.

\section{Consent for publication}

Informed consent was obtained in both written and verbal forms from the patient to publish this case report and any accompanying images.

\section{Competing interests}

The authors declare that they have no competing interests.

Received: 27 November 2020 Accepted: 5 January 2021

Published online: 21 January 2021

\section{References}

1. Rodriguez LV, Terris MK. Risks and complications of transrectal ultrasound guided prostate needle biopsy: a prospective study and review of the literature. J Urol. 1998;160(6 Pt 1):2115-20.

2. de Jesus CM, Correa LA, Padovani CR. Complications and risk factors in transrectal ultrasound-guided prostate biopsies. Sao Paulo Med J = Revista paulista de medicina. 2006;124(4):198-202.

3. Al-Ani HH, Khashram M, Dean A, Bourchier R, Bhamidipaty V, Hill A. Infected abdominal aortic aneurysm after transrectal ultrasound-guided biopsy of the prostate: a report of two cases. Ann Vasc Surg. 2019;61:469. e461-469.e464.

4. Muller BT, Wegener OR, Grabitz K, Pillny M, Thomas L, Sandmann W. Mycotic aneurysms of the thoracic and abdominal aorta and iliac arteries: 
experience with anatomic and extra-anatomic repair in 33 cases. J Vasc Surg. 2001;33(1):106-13.

5. Kyriakides C, Kan Y, Kerle M, Cheshire NJ, Mansfield AO, Wolfe JH. 11-year experience with anatomical and extra-anatomical repair of mycotic aortic aneurysms. Eur J Vasc Endovasc Surg. 2004;27(6):585-9.

6. Grammatico L, Baron S, Rusch E, Lepage B, Surer N, Desenclos JC, Besnier JM. Epidemiology of vertebral osteomyelitis (VO) in France: analysis of hospital-discharge data 2002-2003. Epidemiol Infect. 2008;136(5):653-60.

7. Loeb S, Carter HB, Berndt SI, Ricker W, Schaeffer EM. Complications after prostate biopsy: data from SEER-medicare. J Urol. 2011;186(5):1830-4.

8. Zaytoun OM, Vargo EH, Rajan R, Berglund R, Gordon S, Jones JS. Emergence of fluoroquinolone-resistant Escherichia coli as cause of postprostate biopsy infection: implications for prophylaxis and treatment. Urology. 2011;77(5):1035-41.

9. Taylor AK, Zembower TR, Nadler RB, Scheetz MH, Cashy JP, Bowen D, Murphy AB, Dielubanza E, Schaeffer AJ. Targeted antimicrobial prophylaxis using rectal swab cultures in men undergoing transrectal ultrasound guided prostate biopsy is associated with reduced incidence of postoperative infectious complications and cost of care. J Urol. 2012;187(4):1275-9.

10. Grummet JP, Weerakoon M, Huang S, Lawrentschuk N, Frydenberg M, Moon DA, O'Reilly M, Murphy D. Sepsis and 'superbugs': should we favour the transperineal over the transrectal approach for prostate biopsy? BJU Int. 2014;114(3):384-8.

11. Liss MA, Chang A, Santos R, Nakama-Peeples A, Peterson EM, Osann K, Billimek J, Szabo RJ, Dash A. Prevalence and significance of fluoroquinolone resistant Escherichia coli in patients undergoing transrectal ultrasound guided prostate needle biopsy. J Urol. 2011;185(4):1283-8.

12. Taşdemiroğlu E, Sengöz A, Bagatur E. latrogenic spondylodiscitis. Case report and review of literature. Neurosurg Focus. 2004;16(6):Ecp1.

13. Karapolat $H, A k k o c ̧ Y$, Arda B, Sesli E. Spondylodiscitis caused by sudden onset back pain following transrectal ultrasonography-guided prostate biopsy: a case report. Agri. 2009;21(3):121-5.

14. Kaya M, Kösemehmetoğlu K, Yildirim CH, Orman G, Çelebi Ö, Taşdemiroğlu E. Spondylodiscitis as a spinal complication of transrectal ultrasound-guided needle biopsy of the prostate. Spine. 2012;37(14):E870-872.

15. Dobson G, Cowie CJ, Holliman D. Epidural abscess with associated spondylodiscitis following prostatic biopsy. Ann R Coll Surg Engl. 2015;97(5):e81-82.

16. Hiyama Y, Takahashi S, Uehara T, Ichihara K, Hashimoto J, Masumori N. A case of infective endocarditis and pyogenic spondylitis after transrectal ultrasound guided prostate biopsy. J Infect Chemother. 2016:22(11):767-9.

17. Li CC, Li CZ, Wu ST, Cha TL, Tang SH. Spondylodiscitis with epidural and psoas muscle abscesses as complications after transrectal ultrasoundguided prostate biopsy: report of a rare case. Eur J Case Rep Intern Med. 2017:4(8):000694.

\section{Publisher's Note}

Springer Nature remains neutral with regard to jurisdictional claims in published maps and institutional affiliations.
Ready to submit your research? Choose BMC and benefit from:

- fast, convenient online submission

- thorough peer review by experienced researchers in your field

- rapid publication on acceptance

- support for research data, including large and complex data types

- gold Open Access which fosters wider collaboration and increased citations

- maximum visibility for your research: over $100 \mathrm{M}$ website views per year

At BMC, research is always in progress.

Learn more biomedcentral.com/submissions 